\title{
Temperature-induced abnormalities in sheep oocytes during maturation
}

\author{
R. M. Moor and I. M. Crosby \\ Institute of Animal Physiology, 307 Huntingdon Road, Cambridge CB3 OJQ, U.K.
}

\begin{abstract}
Summary. The susceptibility of sheep oocytes to temperature changes during maturation in vitro was tested by reducing the incubation temperature to $20^{\circ} \mathrm{C}$ at various stages of meiosis. Cooling induced chromosomal abnormalities including disorganized metaphase plates and multipolar spindles in $28-54 \%$ of oocytes cooled at all stages of meiosis from germinal vesicle breakdown (GVBD) to metaphase II. The time of GVBD (8-11 h after the start of culture) was the most sensitive to cooling, whereas fewest abnormalities were found in oocytes cooled in late metaphase I (16-19 h). In addition to the chromosomal abnormalities, unusual vesicles appeared in the cytoplasm of oocytes cooled at $8-11 \mathrm{~h}$ and $12-15 \mathrm{~h}$. No abnormalities in protein synthesis were detected by one-dimensional SDS gel electrophoresis.

The consequences of the abnormalities for the developmental potential of the cooled oocytes were tested by transfer to recipient ewes and fertilization in vivo. After 12 days of development only $6 \%$ and $11 \%$ oocytes cooled at $12-15 \mathrm{~h}$ and $20-23 \mathrm{~h}$ respectively had developed to expanded blastocysts, compared with $44 \%$ of control oocytes.

The results demonstrated that maturing sheep oocytes are very sensitive to a drop in temperature.
\end{abstract}

\section{Introduction}

Mammalian oocytes enter meiosis in early prenatal life and progress to the diplotene stage where they become arrested. The oocytes remain in the germinal vesicle state until they either become reactivated just before ovulation or else undergo degeneration during atresia. The meiotic spindle in reactivated or maturing oocytes consists of two separate groups of components, the chromosomes (and kinetochores) on the one hand and a transient spindle-like structure on the other. It is with this latter set of labile structures and the effects of environmental changes on spindle stability that this paper will be concerned.

It has long been known that the mitotic cycle in somatic cells is prolonged by a reduction in temperature and shortened by an appropriate increase in temperature (Mazia, 1961). One of the most important reasons for these cell-cycle changes can be traced to the temperature sensitivity of the mitotic spindle (see review, Inoue, 1981). That a similar relationship exists in the egg is clear from numerous papers on the embryos of sea urchins, amphibia and other animals (Raff, 1979; Inoue, 1981). The experiments of Rustad (cited by Mazia, 1961) in sea-urchin eggs showed that low temperatures affected not only the rate of chromosome movement but also the physical structure of the mitotic spindle. The reasons for this structure-temperature relationship are now understood and involve microtubules, the main structural elements of spindle fibres (Inoue, 1981). It is clear that low temperatures act by disassembling microtubules and that this disassembly is the result of the depolymerization of the major structure protein of microtubules, tubulin (see Petzelt, 1979).

Our hypothesis, based on the above findings, is that temperature fluctuations induced in mammalian oocytes during maturation adversely affect their subsequent developmental potential. 
Such fluctuations often occur when oocytes are flushed from the follicles of women and animals for in-vitro fertilization and may account for some of the embryonic loss associated with this procedure. The hypothesis has been tested in sheep by cooling oocytes at selected stages during maturation and thereafter examining their nuclear morphology, ability to synthesize proteins and their developmental potential in vivo.

\section{Materials and Methods}

Ovaries were obtained from sheep injected with FSH-CB (horse gonadotrophin fraction CM1 prepared according to Hartree, Mills, Welch \& Thomas, 1968) and slaughtered $24 \mathrm{~h}$ later (Staigmiller \& Moor, 1984). Cumulus-enclosed oocytes were removed from antral follicies $(2.0-5.0 \mathrm{~mm}$ diameter) and matured in vitro using the same culture media and procedures but less gonadotrophin than that used by Staigmiller \& Moor (1984). In outline the technique involves the dissection of cumulus-enclosed oocytes (oocyte complexes) and their culture in Medium M199 supplemented with $10 \%$ heat-inactivated fetal calf serum, gonadotrophin $(5 \mathrm{~g} \mathrm{NIH}-\mathrm{LH} \mathrm{S} 18 / \mathrm{ml}$; $5 \mu \mathrm{g}$ NIH-FSH-S8 $/ \mathrm{ml} ; 20 \mathrm{ng}$ NIH-prolactin-S $8 / \mathrm{ml})$ and oestradiol- $17 \beta(1 \mu \mathrm{g} / \mathrm{ml})$. Additional granulosa cells were harvested from non-atretic follicles and added to the cultures at a concentration of about $5 \times 10^{6}$ cells $/ \mathrm{ml}$ medium. Oocyte complexes (10-20 per $3.5 \mathrm{~mm}$ culture dish) allocated to the control groups were matured for $24 \mathrm{~h}$ at $39^{\circ} \mathrm{C}$ in a system that provided gentle agitation to the medium. Humidity $(95 \%)$ and gas phase $\left(5 \% \mathrm{CO}_{2}\right.$ in air) were carefully controlled throughout culture. The experimental groups were cultured under conditions identical to those of the controls except that the heating elements in the culture system were selectively switched off for $3 \mathrm{~h}$ starting at $8,12,16$ or $20 \mathrm{~h}$ after the beginning of culture.

After culture, most oocytes were fixed, stained with Lacmoid and examined as whole mounts or transferred to recipient ewes (see Moor \& Trounson, 1977, for technical details). Embryos were recovered from the uteri of recipient animals on the 12 th day after transfer and classified as single cell, cleavage arrested or normally expanded blastocysts. The validity of determining developmental potential at this stage of pregnancy has been described in detail elsewhere (Moor \& Trounson, 1977; Staigmiller \& Moor, 1984). As shown in those papers, blastocysts developing normally to Day 12 of pregnancy will, after re-transfer, develop into normal young in $60-70 \%$ of instances.

The remaining groups of oocytes were isotopically labelled with $\left[{ }^{35}\right.$ S]methionine during the final $3 \mathrm{~h}$ of culture. The labelling procedure, electrophoretic separation of polypeptides in individual oocytes and fluorography were all carried out by methods described in detail by Moor, Osborn, Cran \& Walters (1981).

The changes in the temperature of culture medium in the incubator during the cooling and rewarming cycle were measured using a microsensor (RS Components, Corby, Northants, U.K.) immersed in medium in conditions identical to those used for oocyte culture.

\section{Results}

\section{Time-related meiotic changes in extrafollicular oocytes matured in vitro}

In 1965 Dziuk related the maturational changes in ovine oocytes in vivo to time after hCG injection. The results presented in Table 1 complement those data by defining the time-specific sequence of events in 363 sheep oocytes matured in vitro as oocyte-cumulus complexes. The first transition phase starts with chromosome condensation and the beginning of germinal vesicle breakdown (6-8 h after explantation) and culminates with the formation of the first metaphase plate about $2 \mathrm{~h}$ later. The metaphase I stage is prolonged and often persists until $18-20 \mathrm{~h}$ after the initiation of maturation. After a relatively short anaphase I-telophase I transition the extrusion of the 
Table 1. Relationship between the period of maturation in vitro and the meiotic status of cumulusenclosed oocytes cultured after removal from the sheep follicle

\begin{tabular}{cccccccc}
\hline \multirow{2}{*}{$\begin{array}{c}\text { Time from } \\
\text { start of } \\
\text { culture to } \\
\text { fixation (h) }\end{array}$} & $\begin{array}{c}\text { No. of } \\
\text { normal }\end{array}$ & \multicolumn{7}{c}{ Percentage at different stages of meiosis } \\
\cline { 3 - 8 } & oocytes & Dictyate & Prophase & Metaphase I & Anaphase & Telophase & Metaphase II \\
\hline 4 & 26 & 92 & 0 & 8 & 0 & 0 & 0 \\
6 & 14 & 36 & 43 & 21 & 0 & 0 & 0 \\
8 & 32 & 25 & 56 & 19 & 0 & 0 & 0 \\
10 & 28 & 7 & 14 & 71 & 0 & 0 & 7 \\
12 & 32 & 0 & 0 & 81 & 6 & 0 & 13 \\
14 & 32 & 0 & 3 & 88 & 3 & 6 & 0 \\
18 & 104 & 0 & 9 & 44 & 13 & 16 & 18 \\
20 & 24 & 0 & 0 & 83 & 17 & 0 & 0 \\
22 & 51 & 20 & 0 & 50 & 0 & 10 & 20 \\
24 & 24 & 8 & 0 & 33 & 0 & 0 & 58 \\
26 & 47 & 6 & 0 & 13 & 0 & 0 & 81 \\
\hline
\end{tabular}

Table 2. Effect of a $12^{\circ} \mathrm{C}$ temperature reduction at selected intervals during maturation on the nuclear configuration of sheep oocytes examined after a 26 -h maturation period in vitro

\begin{tabular}{cccccccc}
\hline & & \multicolumn{6}{c}{ Nuclear configuration in oocytes after 26-h maturation in vitro(\%) } \\
\cline { 6 - 8 } $\begin{array}{c}\text { Period of cooling } \\
\text { during maturation } \\
\text { (h after LH) }\end{array}$ & $\begin{array}{c}\text { Total } \\
\text { no. of } \\
\text { oocytes }\end{array}$ & Dictyate & Metaphase I & $\begin{array}{c}\text { Anaphase I- } \\
\text { telophase 1 }\end{array}$ & Metaphase II & $\begin{array}{c}\text { Multipolar } \\
\text { spindle }\end{array}$ & $\begin{array}{c}\text { Metaphase } \\
\text { plates }\end{array}$ \\
\hline No cooling & 49 & $3(6)$ & $6(12)$ & 0 & $38(78)$ & 0 & $2(4)$ \\
$8-11$ & 37 & 0 & $4(11)$ & $4(11)$ & $9(24)$ & $8(22)$ & $12(32)$ \\
$12-15$ & 48 & 0 & $14(29)$ & $2(4)$ & $16(33)$ & $4(8)$ & $12(25)$ \\
$16-19$ & 36 & $2(6)$ & $6(17)$ & 0 & $18(50)$ & $2(6)$ & $8(22)$ \\
$20-23$ & 41 & $2(5)$ & $10(24)$ & 0 & $12(29)$ & $5(12)$ & $12(29)$ \\
\hline
\end{tabular}

first polar body and formation of the second metaphase plate occurs and is completed by $24-26 \mathrm{~h}$ after explantation. The periods of cooling were selected to correspond to the major phases of the meiotic cycle. The first group of oocytes was cooled during the GV-metaphase I transition (8-11 h after explantation), the second group represented metaphase I $(12-15 \mathrm{~h})$, the third was cooled during the metaphase I-anaphase I transition (16-19 h) and the fourth group represented the telophase I-metaphase II stage (20-23 h after explantation).

\section{Time-related temperature changes during cooling and rewarming}

Measurements of the temperature of culture medium during three cooling/rewarming cycles showed a mean ( \pm s.e.m.) drop of $12.4 \pm 0.3^{\circ} \mathrm{C}$ in $3 \mathrm{~h}$. The minimum temperature reached was $26.4 \pm 0 \cdot 2^{\circ} \mathrm{C}$. The maximum rate of cooling was $5 \cdot 7^{\circ} \mathrm{C} / \mathrm{h}$. The temperature of the medium rose by $7^{\circ} \mathrm{C}$ within $20 \mathrm{~min}$ of the incubator being switched on again. The rate of increase then slowed and a stable temperature of $38.6 \pm 0 \cdot 2^{\circ} \mathrm{C}$ was reached after $2 \mathrm{~h}$.

\section{Temperature change and nuclear configuration during maturation}

The present results together with those from other studies show that about $80 \%$ of sheep oocyte-cumulus complexes form normal metaphase II plates within $26 \mathrm{~h}$ of culture in our nonstatic system (see Table 2; and Staigmiller \& Moor, 1984). This percentage of metaphase II oocytes is reduced by up to $69 \%$ when the temperature is reduced during maturation. Table 2 shows the 
detailed effects on nuclear configuration of cooling at different stages of the meiotic cycle. A $\chi^{2}$-analysis of the results showed that all the cooled oocytes were significantly different from the controls. Oocytes in the GV-metaphase I transition (8-11 h) were the most susceptible to cooling $\left(\chi^{2}=39 \cdot 5,5\right.$ d.f., $\left.P<0.01\right)$ whilst those at the metaphase I-anaphase I transition $(16-19 \mathrm{~h})$ were least susceptible to temperature fluctuation $\left(\chi^{2}=11.26,5\right.$ d.f., $\left.0.01<P<0.05\right)$. Cooling in the early metaphase I stage $(12-15 \mathrm{~h})$ or during the telephase I-metaphase II transition $(20-23 \mathrm{~h})$ caused almost as many meiotic abnormalities as did cooling during the GV-metaphase I transition.

The characteristic types of chromosomal aberration induced by cooling are illustrated in Plate 1. Figure 1 (Pl. 1) shows a control oocyte matured in vitro with a normal metaphase II configuration. The disorganized 'metaphase' plates shown in Pl. 1, Figs 2 and 3 are the most commonly observed abnormality associated with cooling. Chromosomal clumping (Pl. 1, Fig. 4) and the formation of multipolar spindles (Pl. 1, Figs 5 \& 6) are other characteristic abnormalities induced by temperature fluctuations.

\section{Effect of temperature on polypeptide synthesis and morphological changes in oocytes}

An analysis of the profiles of protein synthesis by SDS gel electrophoresis indicated that no consistent differences can be detected between control oocytes and those cooled at each of the stages tested. A morphological examination of the cytoplasm of cooled oocytes suggested, however, that certain other metabolic events are altered by temperature fluctuations. The most obvious of these cytoplasmic changes occurred in oocytes cooled at the GV-metaphase I transition and during early metaphase I. The characteristic lipid-like vesicles that accumulate in many oocytes cooled between 8 and $15 \mathrm{~h}$ after explantation are shown in Pl. 1, Fig. 2. The vesicles, which have not yet been chemically identified, did not appear in oocytes cooled at the metaphase I-anaphase I or telephase I-metaphase II transitions.

\section{Temperature change and developmental potential of oocytes}

Embryo transplantation followed by an analysis of all resultant embryos on Day 12 or later represents the only current means of determining developmental potential of treated oocytes. The results presented in Table 3 summarize the effects of cooling oocytes on subsequent embryonic development. Control oocytes matured in vitro developed into normal blastocysts in $44 \%$ of instances, a slightly lower rate of development than that obtained previously (Staigmiller \& Moor, 1984). Cooling during early metaphase I $(12-15 \mathrm{~h})$ decreased to only $6 \%$ the number of oocytes developing subsequently into normal blastocysts. By comparison, a slightly higher percentage ' $(11 \%)$ of oocytes cooled at the telophase I-metaphase II transition (20-23 h) developed normally after transfer.

Table 3. Effect of a $12^{\circ} \mathrm{C}$ temperature reduction at selected times during oocyte maturation on subsequent embryonic development of sheep at Day 12

\begin{tabular}{lcccrc}
\hline & & \multicolumn{3}{c}{$\begin{array}{c}\text { Embryonic development } \\
\text { (\% of no. recovered in parentheses) }\end{array}$} \\
\cline { 3 - 5 } $\begin{array}{c}\text { Cooling period } \\
\text { (h after LH) }\end{array}$ & $\begin{array}{c}\text { No. of oocytes } \\
\text { transferred }\end{array}$ & $\begin{array}{c}\text { No. of embryos } \\
\text { recovered }\end{array}$ & $\begin{array}{c}\text { Single } \\
\text { celled }\end{array}$ & $\begin{array}{c}\text { Arrested } \\
\text { cleavage }\end{array}$ & Blastocysts \\
\hline No cooling & 87 & 68 & $37(54)$ & $2(3)$ & $30(44)$ \\
$12-15$ & 76 & 47 & $43(91)$ & $1(2)$ & $3(6)$ \\
$20-23$ & 129 & 85 & $65(76)$ & $10(12)$ & $10(12)$ \\
\hline
\end{tabular}


PLATE 1
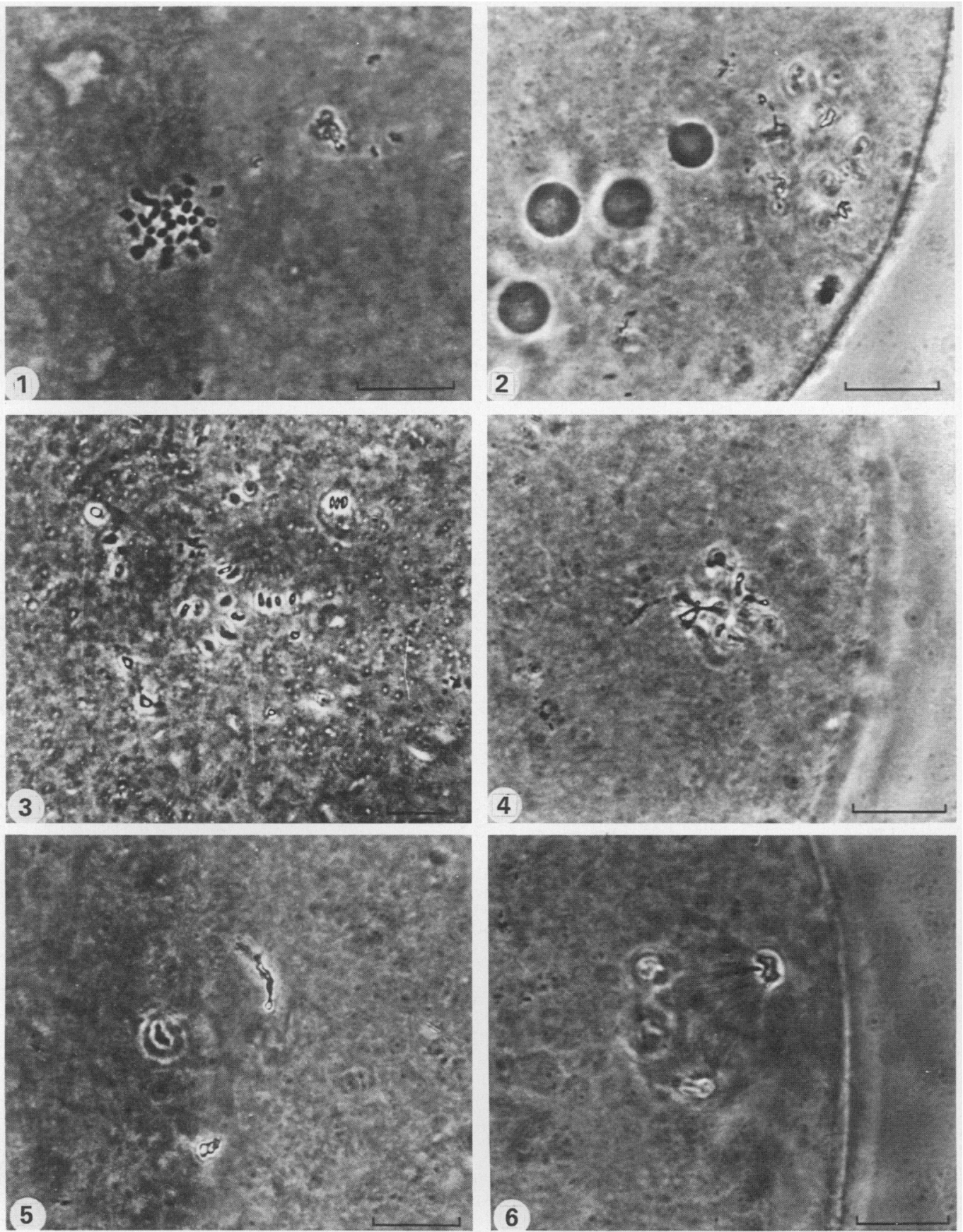

Nuclear configurations of sheep oocytes cooled at various times during maturation in vitro. Phasecontrast micrographs of oocytes stained with Lacmoid. Scale bars are $20 \mu \mathrm{m}$ in length.

Fig. 1. Control oocytes, no cooling. Normal metaphase II configuration.

Fig. 2. Oocyte cooled at $8-11 \mathrm{~h}$. disorganized chromosomes.

Fig. 3. Oocyte cooled at $2023 \mathrm{~h}$. disorganized chromosomes.

Fig. 4. Oocyte cooled at $811 \mathrm{~h}$. clumped chromosomes.

Figs 5 and 6. Oocytes cooled at $12-15$ h. multipolar spindles.

(Facing p. 470) 


\section{Discussion}

The studies described in this report demonstrate the sensitivity of sheep oocytes to temperature changes during meiosis. This sensitivity is expressed at a morphological level, in terms of the disorganization of the chromosomes and the appearance of unusual vesicles in the cytoplasm, but more importantly in the greatly reduced ability of the cooled oocytes to develop normally after fertilization in vivo. The method chosen was designed to subject the oocytes to a minimum of temperature stress by cooling them slowly. Because the oocytes remained otherwise undisturbed, there was no possibility that changes in $\mathrm{pH}$ or osmolarity could account for the observed effects on morphology and development.

Oocytes cooled below $29^{\circ} \mathrm{C}$ for $3 \mathrm{~h}$ at various stages of meiotic maturation exhibited chromosomal defects including disorganized metaphase plates, clumping of chromatin and the formation of multipolar spindles. Moreover, only 6-11\% of cooled oocytes developed to blastocysts after 12 days, compared with $44 \%$ of control oocytes. These results complement those in two earlier reports describing the generation of meiotic abnormalities in mouse oocytes subjected to temperature shock. Karp \& Smith (1975) reported that $46.4 \%$ of oocytes cooled to $23^{\circ} \mathrm{C}$ had abnormal numbers of chromosomes; the rate of induced aneuploidy was estimated to be $19 \%$ and $5 \%$ of oocytes were apparently diploid as a result of failure to extrude the first polar body. Baumgartner \& Chrisman (1981) collected ovulated eggs from mice subjected to an environmental temperature of $35^{\circ} \mathrm{C}$. Meiosis was disrupted in more than $25 \%$ of these oocytes; $12 \%$ were at metaphase I and $13 \%$ exhibited fragmented or arrested germinal vesicles.

The disturbance of oocyte meiosis by low temperatures is most likely to be a consequence of the disruption of the meiotic spindle. The spindle is an extremely labile structure composed of a highly organized array of microtubules which are assembled by the polymerization of a preexisting pool of the principal constituent protein, tubulin (see Inoue, 1981, for a review on spindle structure and properties). A dynamic equilibrium exists between polymerized microtubules and tubulin subunits, the balance of which is shifted towards disassembly by low temperature. Observations of mitotically-dividing living cells under the light microscope have revealed that chilling results in the disassembly of the spindle fibres within minutes, followed by the equally rapid reassembly of the spindle after a return to normal temperature (Inoue, 1981).

The production of a spindle capable of bringing about such an ordered process as chromosome segregation clearly requires that microtubule assembly takes place only at specialized foci within the cell, collectively known as microtubule-organizing centres. The principal centres in the oocyte are at the poles of the spindle. Microtubules are also attached to the kinetochores of the chromosomes and although they are probably not assembled from here, kinetochore-associated tubules may be more stable and cold-resistant than unattached tubules (McIntosh, 1984).

Although reassembly of microtubules is rapid once the normal temperature is restored, there are a number of possible ways in which errors can occur, resulting in the formation of abnormal spindles similar to those described in this report. Firstly, chromosomes may rotate during cooling and after reattachment at the kinetochore may face towards the wrong pole of the spindle (Henderson, Nicklas \& Koch, 1970). This will result in migration of the chromosome in the wrong direction, and hence aneuplody. Alternatively, fragmentation of the polar microtubule-organizing centres may occur as a result of cooling. This leads to the formation of a multipolar spindle, as observed in our experiments.

Although it is more difficult to explain the cytoplasmic abnormalities observed in cooled oocytes in this study, it is possible that these too may be related to microtubule disassembly. Maturation involves the relocation of cellular organelles, for example, the migration of cortical granules to the periphery, the aggregation of mitochondria and a general accumulation of mitochondria, vesicles and lipid droplets in the middle of the oocyte as maturation proceeds (Kruip, Cran, van Beneden \& Dieleman, 1983; Cran, 1985). Disruption of the cytoskeleton as a result of tubulin depolymerization would be expected to interfere with these intracellular move- 
ments. Clearly, a more detailed study of organelle distribution in cooled oocytes is necessary before any conclusions can be reached.

As described above, the selection of cooling times was designed to correspond to different stages in meiosis. The temperature sensitivity of the rate of the various phases of meiosis is well documented (see Mazia, 1961), but there seems to be little information on the relative sensitivities to disruption by cooling. Our analysis of meiotic abnormalities suggests that the time of GVBD is the most sensitive and the metaphase I-anaphase I transition the least sensitive to cooling. Unfortunately these stages were not selected for analysis of developmental potential when the experiments were planned so no confirmation was provided by transfer of these oocytes.

It might intuitively be expected that meiosis would be most sensitive to disruption at the time of chromosome attachment to the spindle and again during segregation of the chromosomes in anaphase/telophase. In contrast, the long metaphase period, when there is little apparent action, might be expected to be less sensitive to spindle disassembly. Although our results broadly support this expectation, the 16-19 h (metaphase I-anaphase I) oocytes showing the least degree of disruption, there is also strong evidence that the time of GVBD is a particularly sensitive period in oocyte maturation. The results of other experiments have shown that the oocyte undergoes a dramatic reprogramming at this time, involving changes in protein synthesis, protein phosphorylation and membrane transport (Moor \& Smith, 1979; Moor et al., 1981; Crosby, Osborn \& Moor, 1984). Therefore, the susceptibility of the oocyte to temperature change at this time may not be entirely, or indeed primarily, due to nuclear events.

The ability of exposure to room temperature to reduce drastically the developmental competence of oocytes is relevant in the handling of human oocytes recovered from women during in-vitro fertilization therapy. Such oocytes are typically at the later stages of the first meiotic division and, therefore, might be expected to be particularly susceptible to cold shock. Although efforts are made to maintain the oocytes at $37^{\circ} \mathrm{C}$, the necessity to search for them under the microscope before they are put into culture must inevitably lead to a drop in temperature. Our own observations show that the temperature of medium in a small culture dish drops by about $1{ }^{\circ} \mathrm{C} / \mathrm{min}$ when the dish is placed on an unheated surface. Given the present protocol used for obtaining oocytes for in-vitro fertilization it is difficult to see how this problem can be entirely overcome. However, every effort should be made to keep the oocytes at $37^{\circ} \mathrm{C}$ throughout recovery.

We thank Mr Colin Brown for the preparation of the horse gonadotrophin used for treatment of experimental animals. Ovine gonadotrophins were a gift from the National Institute of Arthritis, Diabetes and Digestive \& Metabolic Diseases, Bethesda, Maryland, U.S.A.

\section{References}

Baumgartner, A.P. \& Chrisman, C.L. (1981) Cytogenetic analysis of ovulated mouse oocytes following hyperthermic stress during meiotic maturation. Expl Cell Res. 132, 359-366.

Cran, D.G. (1985) Qualitative and quantitative structural changes during pig oocyte maturation. J. Reprod. Fert. 74, 237-245.

Crosby, I.M., Osborn, J.C. \& Moor, R.M. (1984) Changes in protein phosphorylation during the maturation of mammalian oocytes in vitro. $J$. exp. Zool. 229, 459-466.

Dziuk, P.J. (1965) Timing of maturation and fertilization of the sheep egg. Anat. Rec. 153, 211-223.

Hartree, A.S., Mills, J.B., Welch, R.A.S. \& Thomas, M. (1968) Fractionation of protein hormones from horse pituitary glancis. J. Reprod. Fert. 17, 291-303.
Henderson, S.A., Nicklas, R.B. \& Koch, C.A. (1970) Temperature-induced orientation instability during meiosis: an experimental analysis. J. Cell Sci. 6, $323-350$.

Inoue, S. (1981) Cell division and the mitotic spindle. $J$. Cell Biol. 91, 131s-147s.

Karp, L.E. \& Smith, W.D. (1975) Experimental production of aneuploidy in mouse oocytes. Gynecol. Invest. 6, 337-341.

Kruip, T.A.M., Cran, D.G., van Beneden, T.H. \& Dieleman, S.J. (1983) Structural changes in bovine oocytes during final maturation in vivo. Gamete Res. 8, 29-47.

Mazia, D. (1961) Mitosis and the physiology of cell division. In The Cell, Vol. 3, pp. 77-412. Eds J. Brachet \& A. Mirsky. Academic Press, New York. 
McIntosh, J.R. (1984) Mechanisms of mitosis. Trends in Biochem. Sci. 100, 195-198.

Moor, R.M. \& Smith, M.W. (1979) Amino acid transport in mammalian oocytes. Expl Cell Res. 119, 333-341.

Moor, R.M. \& Trounson, A.O. (1977) Hormonal and follicular factors affecting maturation of sheep oocytes in vitro and their subsequent developmental capacity. J. Reprod. Fert. 49, 101-109.

Moor, R.M., Osborn, J.C., Cran, D.G. \& Walters, D.E. (1981) Selective effect of gonadotrophins on cell coupling, nuclear maturation and protein synthesis in mammalian oocytes. J. Embryol. exp. Morph. 61, 347-365.
Petzelt, C. (1979) Biochemistry of the mitotic spindle. Int. Rev. Cytol. 60, 53-85.

Raff, E.C. (1979) The control of microtubule assembly in vivo. Int. Rev. Cytol. 59, 1-85.

Staigmiller, R.B. \& Moor, R.M. (1984) Effect of follicle cells on the maturation and developmental competence of ovine oocytes matured outside the follicle. Gamete Res. 9, 221-229.

Received 16 January 1985 